\title{
Linfangioma quístico retroperitoneal adherido a cabeza pancreática*
}

\author{
Drs. JAIME RUIZ-TOVAR ${ }^{1}$, EMILIO RIPALDA ${ }^{1}$, TERESA MARTÍN ${ }^{1}$, \\ RAFAEL BENI ${ }^{1}$, JAVIER NUÑO ${ }^{1}$ \\ 1 Servicio de Cirugía General y del Aparato Digestivo. \\ Hospital Universitario Ramón y Cajal, Madrid, España.
}

\begin{abstract}
\section{Cystic retroperitoneal lymphangioma}

Cystic retroperitoneal lymphangioma is an unfrequent pathology. Most cases are asymptomatic, and when symptomatic, they tend to present as abdominal pain and bowel obstruction secondary to compression of the bowel loops. Treatment of choice is surgical excision. We present a case of cystic retroperitoneal lymphangioma with adhesions to the second portion of duodenum and head of the pancreas. Once separated from the pancreas, it remained denudated. To avoid an eventual pancreatic fistula, Tachosil@ was placed as sealing method. Postoperative course was uneventful.
\end{abstract}

Key words: Cystic retroperitoneal lymphangioma, cystic retroperitoneal lesions, Tachosil@.

\section{Resumen}

El linfangioma quístico retroperitoneal es una patología de poca frecuencia. La mayoría de los casos son asintomáticos y cuando presentan síntomas, lo más frecuente es dolor abdominal u obstrucción intestinal por compresión de asas intestinales. Su tratamiento consiste en la extirpación completa de la lesión. Presentamos un caso de linfangioma quístico retroperitoneal adherido a $2^{\mathrm{a}}$ porción duodenal y cabeza pancreática. Al separarlo del tejido pancreático, quedó un borde cruento. Para evitar una fístula pancreática, se colocó una placa de Tachosil ${ }^{\circledR}$ a modo de sellante. No aparecieron complicaciones postoperatorias.

Palabras clave: Linfangioma quístico retroperitoneal, lesiones quísticas retroperitoneales, Tachosil@.

\section{Introducción}

El linfangioma quístico retroperitoneal es una patología muy poco frecuente, suponiendo aproximadamente el $1 \%$ de todos los linfangiomas, habiendo menos de 200 casos descritos en la literatura ${ }^{1,2}$, descubierto de forma incidental durante la realización de alguna prueba de imagen por otro motivo. Cuando son sintomáticos, lo más frecuente es el dolor abdominal, seguido de la obstrucción intestinal por compresión de asas de intestino delgado; un sangrado o infección del quiste puede provocar un cuadro de abdomen agudo ${ }^{3,4}$.

\footnotetext{
*Recibido el 25 de Julio de 2009 y aceptado para publicación el 9 de Octubre de 2009.

Correspondencia: Dr. Jaime Ruiz-Tovar

Corazón de María, 64, $7^{\circ} \mathrm{J}$

28002 - Madrid, España.

E-mail: jruiztovar@gmail.com
} 


\section{Caso clínico}

Varón de 39 años, completamente asintomático y sin antecedentes personales de interés, es sometido a un reconocimiento rutinario de empresa y en una ecografía abdominal se observa una imagen quística septada de $6 \mathrm{~cm}$ de diámetro en el espacio anterior al riñón derecho. Se realiza una TC abdominal, objetivándose una lesión heterogénea de naturaleza indeterminada, en espacio pararrenal anterior derecho, que se extiende a la región anterior y lateral de la cava inferior. La Resonancia Magnética abdominal determinó la presencia de una lesión de $10 \mathrm{~cm}$ multiloculada con septos finos sin signos de infiltración sobre estructuras vecinas (cava, hígado, riñón, duodeno, páncreas), sugestiva de linfangioma retroperitoneal. Se decidió intervenir al paciente, observándose durante la cirugía una tumoración retroperitoneal de posible origen linfático con contenido quiloso, adherida a $2^{\mathrm{a}}$ porción duodenal y cara anterior de cabeza pancreática (Figura 1). Tras realizar una maniobra de Kocher, se liberó el tumor de la cara posterior de cabeza pancreática, marco duodenal, cara anterior pancreática y meso del colon derecho, preservando vasos cólicos derechos y pancreáticos. El tumor estaba íntimamente adherido a la cara anterior de la cabeza pancreática y para poder resecarlo completamente, fue necesario dejar un borde cruento en ella. Se decidió la colocación de una esponja de TachoSil ${ }^{\circledR}$ en el lecho cruento de páncreas, para intentar sellar la zona y evitar una fístula (Figura 2). Se dejó un drenaje tipo Blake -Drain $\mathrm{n}^{\mathrm{o}} 19$ en el lecho pancreático. El postoperatorio fue correcto. Se midieron los niveles de amilasa sérica y en el líquido de drenaje al $3^{\circ}$ día, siendo ambos normales. El paciente fue dado de alta al $6^{\circ}$ día de la intervención.

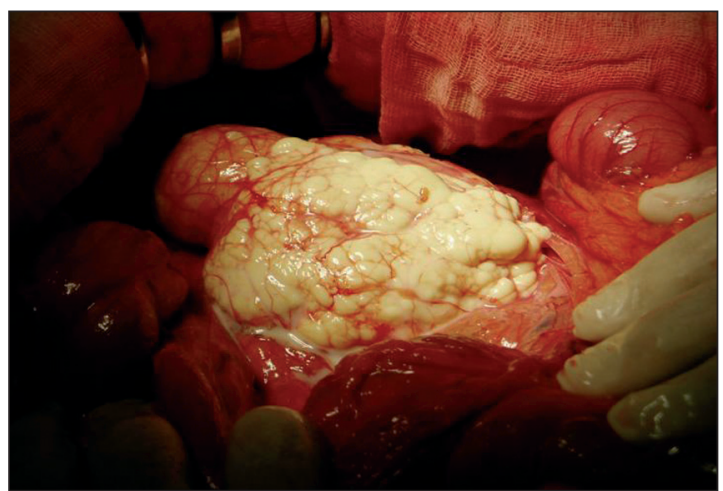

Figura 1: Tumoración retroperitoneal de posible origen linfático con contenido quiloso, adherida a $2^{\mathrm{a}}$ porción duodenal y cara anterior de cabeza pancreática.

\section{Discusión}

El linfangioma quístico retroperitoneal es una patología muy poco frecuente, suponiendo aproximadamente el $1 \%$ de todos los linfangiomas, habiendo menos de 200 casos descritos en la literatura ${ }^{1,2}$. La mayoría de los casos son asintomáticos, descubierto de forma incidental durante la realización de alguna prueba de imagen por otro motivo. Cuando son sintomáticos, lo más frecuente es el dolor abdominal, seguido de la obstrucción intestinal por compresión de asas de intestino delgado. Un sangrado o la infección del quiste puede provocar un cuadro de abdomen agudo ${ }^{3,4}$.

Alcanzar el diagnóstico preoperatorio de linfangioma retroperitoneal es raro. Las pruebas de imagen más empleadas son la ecografía, que permite identificar la naturaleza quística de la lesión, la tomografía computada y la resonancia magnética, que informan acerca del tamaño, extensión y relación con estructuras vecinas ${ }^{5}$. La punción no suele realizarse por la localización de la lesión y el riesgo de diseminación tumoral ${ }^{6}$. Se debe establecer un diagnóstico diferencial con cualquier entidad que pueda manifestarse como una masa quística retroperitoneal, incluyendo patologías malignas como tumores quísticos pancreáticos, metástasis ganglionares retroperitoneales, sarcomas retroperitoneales, teratomas, metástasis quísticas de tumores gástricos u ováricos, mesenquimoma maligno, mesotelioma quístico o cistoadenocarcinoma biliar, y patologías benignas como hematomas, linfoceles secundarios a cirugías previas, abscesos, adenoma microquístico pancreático, quistes uroteliales o quistes originarios del intestino anterior primitivo ${ }^{1,5,7}$.

El tratamiento consiste en la extirpación completa de la lesión, con lo que se consigue un buen

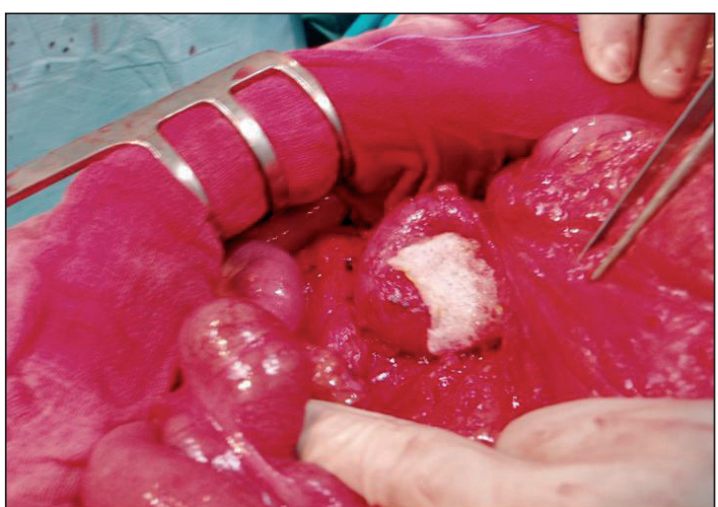

Figura 2: Colocación de una esponja de TachoSil® en el lecho cruento en cabeza pancreática, para intentar sellar la zona y evitar una fístula pancreática. 
pronóstico. Las resecciones agresivas sacrificando estructuras vitales no son justificables, dada la naturaleza benigna de la lesión. Sin embargo, las extirpaciones incompletas pueden conllevar la aparición de recidivas ${ }^{8}$.

TachoSil® es una esponja formada por un soporte de colágeno con fibrinógeno y tromabina humanos en la superficie, proporcionando así una hemostasia rápida y eficaz ${ }^{9}$. Debido a su estructura en forma de panal de abeja, permite un sellado hermético a fluidos y a aire, habiendo sido utilizado con éxito como sellante en pulmón, evitando fugas aéreas ${ }^{10}$, en vejiga ${ }^{11}$ e incluso en anastomosis gastrointestinales ${ }^{12}$, previniendo fístulas líquidas. Gracias a las propiedades hemostáticas y sellantes descritas, TachoSil ${ }^{\circledR}$ está ya usándose con gran éxito en cirugía hepática, pulmonar y renal, e incluso en cirugía pancreática con fines hemostáticos. Con este caso queremos mostrar que TachoSil ${ }^{\circledR}$ puede contribuir a una menor formación de fístulas pancreáticas, una de las principales complicaciones de la cirugía del páncreas.

\section{Referencias}

1. Cherk M, Nikfajam M, Christophi C. Retroperitoneal lymphangioma. Asian J Surg 2006; 29: 51-54.

2. Hauser H, Mischinger HJ, Beham A, Berger A, Cerwenka H, Razmara J, et al. Cystic retroperitoneal lymphangiomas in adults. Eur J Surg Oncol 1997; 23: 322-326.

3. Enzinger FM, Weiss SW. Soft tissue tumours, $3^{\text {rd }}$ edition. St Louis: Mosby, 1995.

4. Takiff H, Calabria R, Yin L, Stabile BE. Mesenteric cysts and intra-abdominal cystic lymphangiomas. Arch Surg 1985; 12: 1266-1269.

5. Arzoz Fabregas M, Ibarz Servio L, Areal Calama J, González Satué C, Ruiz Domínguez J, Saladié Roig JM. Linfangioma quístico, nuestra experiencia. Actas Urol Esp 2006; 30: 723-727.

6. Hayami S, Adachi Y, Ishigooka M, Suzuki H, Sasagawa I, Kubota Y, et al. Retroperitoneal cystic lymphangioma diagnosed by computerized tomography, magnetic resonance imaging and thin needle aspiration. Int Urol Nephrol 1996; 28: 21-26.

7. Nuzzo G, Lemmo G, Marrocco-Trischitta MM, Boldrini G, Giovannini I. Retroperitoneal cystic lymphangioma. J Surg Oncol 1996; 61: 234-237.

8. Su CM, Yu MC, Chen HY, Tseng JH, Jan YY, Chen MF. Single-centre results of treatment of retroperitoneal and mesenteric cystic lymphangiomas. Dig Surg 2007; 24: 181-185.

9. Siemer S, Lahme S, Altziebler S, Machtens S, Strohmaier W, Wechsel HW, et al. Efficacy and safety of TachoSil as haemostatic treatment versus standard suturing in kidney tumour resection: a randomised prospective study. Eur Urol 2007; 52: 1156-1163.

10. Anegg U, Lindenmann J, Matzi V, Smolle J, Maier A, Smolle-Jüttner F. Efficiency of fleece-bound sealing

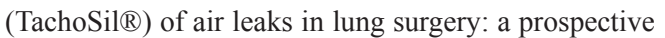
randomised trial. Eur J Cardiothorac Surg 2007; 31 : 198-202.

11. Erdogru T, Sanlı A, Celik O, Baykara M. Laparoscopic transvesical repair of recurrent vesicovaginal fistula using with fleece-bound sealing system. Arch Gynecol Obstet 2008; 277: 461-464.

12. Nordentoft T, Rømer J, Sørensen M. Sealing of gastrointestinal anastomoses with a fibrin glue-coated collagen patch: a safety study. J Invest Surg 2007; 20: 363-369. 